\title{
Comparison of Clinical and Neuroimaging Characteristics of Congenital and Possible Congenital Cytomegalovirus Infected Infants: A Retrospective Study
}

\author{
Nuriye Tarakcı ${ }^{1}$ Cahide Bulut $^{2}$ Hüseyin Çaksen ${ }^{1}$ \\ ${ }^{1}$ Department of Pediatrics, Meram Medical Faculty, Necmettin \\ Erbakan University, Konya, Turkey \\ ${ }^{2}$ Department of Pediatrics, Batman Child Hospital, Konya, Turkey \\ ${ }^{3}$ Department of Pediatrics, Medova Hospital, Konya, Turkey
}

\author{
Rahmi Örs ${ }^{3}$
}

J Pediatr Infect Dis 2019;14:89-95.

\begin{abstract}
Address for correspondence Nuriye Tarakcı, MD, Department of Pediatrics, Meram Medical Faculty, Necmettin Erbakan University, 42080 Konya, Turkey (e-mail: nuriyetarakci@hotmail.com).
\end{abstract}

\begin{abstract}
Keywords

- congenital CMV

- possible congenital CMV

- neuroimaging

Background To evaluate clinical and neurodevelopmental outcomes and neuroimaging results of patients diagnosed with congenital cytomegalovirus (cCMV) infection and those diagnosed with possible congenital CMV (possible cCMV) infection.

Methods From January 2014 to January 2016, 24 patients were diagnosed with congenital CMV infection. Clinical and neuroimaging results of these patients were examined from their medical records.

Results Of the 24 patients, 6 were diagnosed with cCMV, whereas 18 patients were diagnosed with possible cCMV. Petechia was the most common symptom observed in patients with CCMV infection, whereas microcephaly was more common in patients with possible cCMV. Of the 24 patients, 5 (20.8\%) showed normal neuroimaging characteristics. The most commonly encountered abnormal findings in brain magnetic resonance imaging were hypoplasia of corpus callosum (16.7\%) and hydrocephalous (16.7\%). All of the patients had poor neurodevelopmental outcomes.

Conclusions Early diagnosis of asymptomatic CCMV infection is difficult. This study suggests that possible cCMV infections are more prevalent compared with cCMV infections, and delays in diagnosis occur due to not keeping suspicion of CMV infection in early phase. Clinicians should consider long-term neurodevelopmental sequelae of cCMV and possible cCMV infections and remember to screen congenital CMV infection in suspected individuals.
\end{abstract}

\section{Introduction}

Neonatal congenital infections are an important cause of mortality and morbidity including long-term neurodevelopmental sequelae. Cytomegalovirus (CMV) is the most common cause of congenital viral infections and accounts for 0.5 to $1 \%$ live births in developed countries. ${ }^{1}$ In the majority (90\%) of infants with congenital CMV (cCMV) infection, no clinical finding is encountered during neonatal period (asymptomatic

received

June 6, 2018

accepted after revision

October 9, 2018

published online

November 18, 2018

infection). However, $10 \%$ of the patients can be diagnosed with symptoms including disseminated disease and/or central nervous system involvement (symptomatic infection). ${ }^{2}$

Many pathological and anatomical alterations, such as ventriculomegaly, microcephaly, cortical anomalies, calcifications, subependymal cysts, and cerebellar hypoplasia can be detected by magnetic resonance (MR) imaging, although they are not pathognomonic for congenital CMV infection. However, these MR findings are present also in congenital

Copyright @ 2019 by Georg Thieme Verlag KG, Stuttgart · New York
DOI https://doi.org/ 10.1055/s-0038-1675765. ISSN 1305-7707. 
central nervous system (CNS) infections, developmental anomalies of brain (for instance, lissencephaly, pachygyri), leukoencephalopathy and cerebral palsy, and differential diagnosis should be made.

In previous studies, poor neurodevelopmental outcomes such as hearing loss, loss of vision, chorioretinitis, microcephaly, cognitive disorders, and cerebral palsy were reported in infants with congenital CMV infection who were whether symptomatic or asymptomatic and developed the findings within the 1 st year of life. ${ }^{3,4}$ Nevertheless, it is still controversial to predict which babies will have a developmental disorder. In the literature, there are studies concerning computed tomography $(\mathrm{CT})$ images showing destructive brain lesions associated with poor neurological outcomes ${ }^{5}$ and MR images showing white matter and migration abnormalities ${ }^{4}$ in patients with congenital CMV infection.

In this study, we described clinical characteristics and brain neuroimaging findings of patients with symptomatic congenital CMV infection and patients who were asymptomatic at birth and diagnosed with possible cCMV within the 1 st year of life. Our objective is to find different clinical presentations of congenital CMV infection and to remind clinicians to be aware of the damage that CMV causes in the brain.

\section{Methods}

Infants, under 1 year of age, who were admitted to Konya Necmettin Erbakan University Meram Faculty of Medicine Pediatric Neurology clinic between January 2014 and January 2016 and had microcephaly, hydrocephalous, hearing loss, seizures, chorioretinitis, and structural and/or white matter-migration abnormalities on brain MR images were included in the study. The study was approved by Necmettin Erbakan University Ethics Committee.

The cCMV infection was defined as detection of CMV in urine by polymerase chain reaction (PCR) within the first 3 weeks of life. Symptomatic and asymptomatic cCMV infections were defined as presence and/or absence of the following symptoms or findings within the first 30 days of life which may be attributed to cCMV: conjugated hyperbilirubinemia, petechia, hepatosplenomegaly, thrombocytopenia, chorioretinitis, intrauterine growth retardation, microcephaly, seizures, or intracranial calcifications. ${ }^{6}$ Possible cCMV infection was defined as meeting of all of the following criteria after the first 3 weeks of life, including (1) presence of one or more signs of congenital CMV, (2) exclusion of other conditions that may lead to these abnormalities (3) detection of CMV in urinary or salivary samples by viral culture or PCR from the 3rd week of life to 1 year of age. ${ }^{7}$

The following data were obtained retrospectively from patient records and the electronic medium: age, gender, symptoms, presence of hearing loss, CMV DNA, PCR results, and neuroimaging findings. In all of patients, sensorineural hearing loss was measured again with the brainstem-evoked response testing when they received a cCMV and possible cCMV diagnosis, even if neonatal hearing screening was normal. Patients' prenatal, natal, and postnatal histories, as well as physical examination findings were evaluated.
Patients with negative urinary CMV PCR result, intrauterine toxin exposure, postnatal blood transfusion, and history of genetic and/or metabolic disease were excluded.

All patients underwent a urinary CMV DNA PCR test. The samples were analyzed by real time PCR using a commercial kit (Qiagen, Germany) according to the manufacturer's instructions. All had positive results. For the patients for whom possible congenital CMV infection was suspected, the following tests were performed for differential diagnosis: Tandem mass spectrometry, plasma and urinary amino acid analysis, urinary organic acid analysis, other viral markers, karyotype analysis, and for individuals with suspected Rett's syndrome, MECP2 mutation.

Findings regarding follow-up examinations were also recorded. Neurodevelopmental outcomes of all patients included in the study were evaluated by using Denver Developmental Screening Test II. Cranial imaging, results such as ultrasound (US), CT, and MR which were performed at the time of diagnosis and during follow-up, were recorded.

\section{Results}

Of the 24 patients diagnosed with cCMV and possible cCMV infections, 6 (25\%) were diagnosed with CCMV and 18 patients (75\%) were diagnosed with possible cCMV infection. Patients' female/male ratio was 9/15. Demographic characteristics and clinical findings of these patients are shown in -Table 1. Of the 24 patients included in the study, 6 (25\%) had history of admission in neonatal intensive care unit after birth and almost all were diagnosed with cCMV infection. Petechia was the most common symptom in cCMV infection. In this group, the disease was observed to progress clinically poor and $50 \%(3 / 6)$ of the patients died within the first few days of life. Only one of the six patients included in this group had brain MR image and this situation arose from the fact that brain MR images could not be performed just because approximately half of patients died within the 1st week of life. The most common symptom in cases of possible cCMV infection was microcephaly (61\%). Other common clinical findings encountered in this group were as follows: seizure (50\%), hydrocephalous (22\%), loss of vision (16\%), hearing loss $(11 \%)$, jaundice (5.5\%; - Table 1 ).

Of the 24 patients included in the study, $5(20.8 \%)$ had normal neuroimaging characteristics. Cranial calcification was determined in 25\% (6/24) of the cases. Calcifications were detected by cranial US (in two patients) and cranial CT (in four patients). Abnormal brain MR image findings of these patients included hypoplasia of corpus callosum (16.7\%), hydrocephalous (16.7\%), lissencephaly (12.5\%), intracranial hemorrhage (12.5\%), colpocephaly $(8.3 \%)$, delayed mineralization $(8.3 \%)$, mega cisterna magna (4.2\%), cerebral-cerebellar atrophy (4.2\%), and type 2 Chiari malformation (4.2\%; - Table 2, - Fig. 1).

Craniosynostosis was detected in three patients by cranial CT and three-dimensional CT (-Fig. 2; - Table 2). Neurodevelopmental developments of all patients included in the study, evaluated by using Denver Developmental Screening Test II, were determined to be below the normal range for their age groups. 


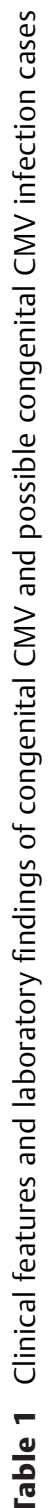

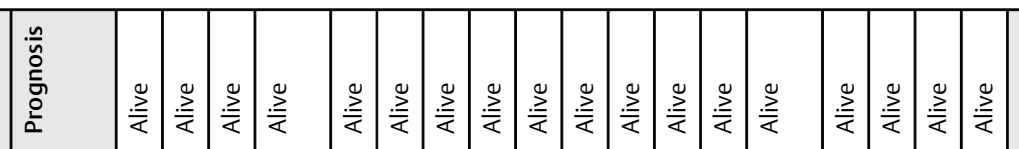

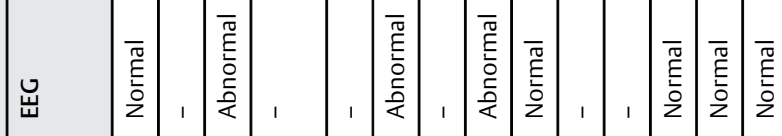

$+\frac{1}{3}$

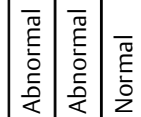
.

离

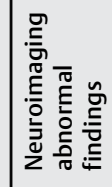

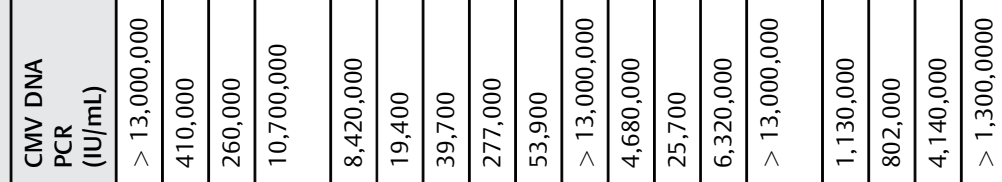

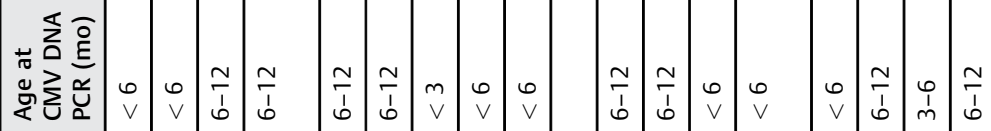

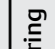

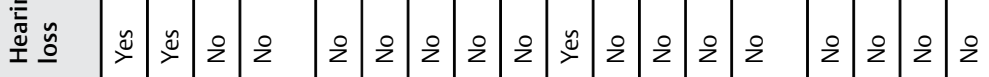

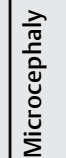

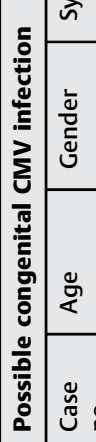

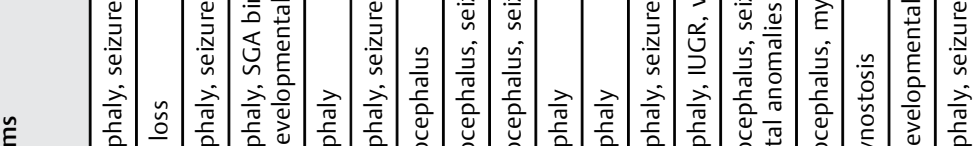

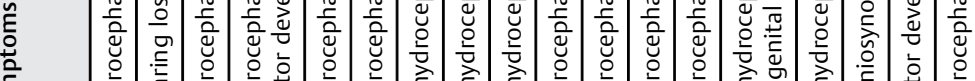

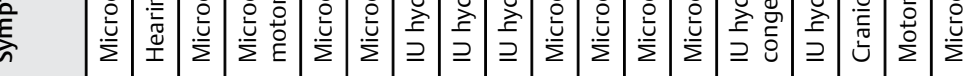
这

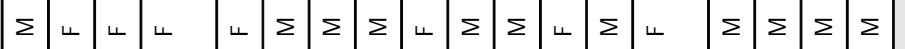

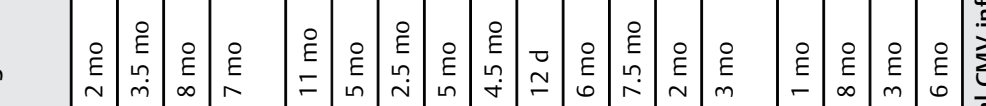
$\dot{\leftrightarrow}$
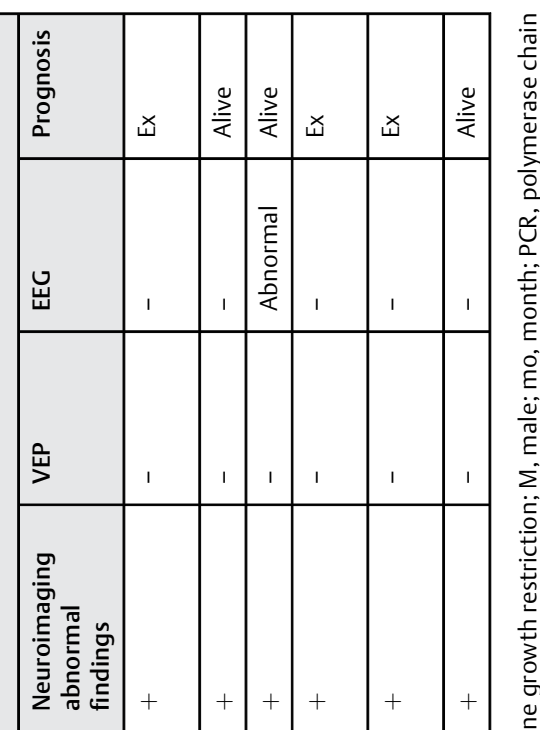

芦

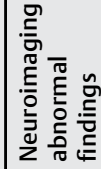

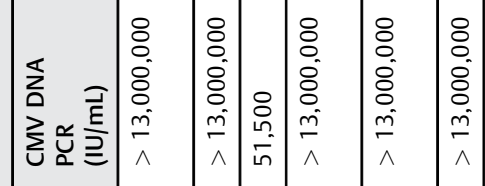

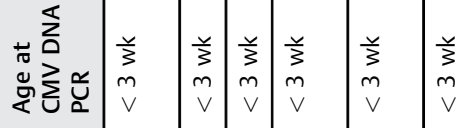

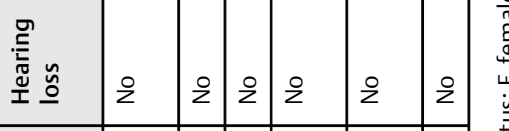

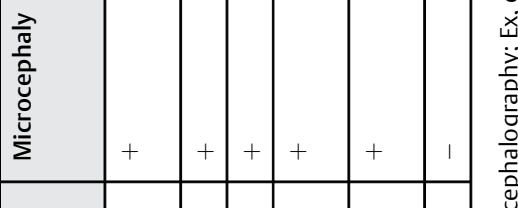

若

空

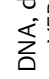


Table 2 Neuroimaging findings of congenital CMV and possible congenital CMV infection cases

\begin{tabular}{|c|c|c|c|c|c|}
\hline $\begin{array}{l}\text { Case } \\
\text { no }\end{array}$ & Cranial US & $\mathrm{CT}$ & $\begin{array}{l}\text { Cranial } \\
\text { MRI }\end{array}$ & $\begin{array}{l}\text { Temporal } \\
\text { bone MRI }\end{array}$ & Three-dimensional CT \\
\hline 1 & - & Lissencephaly & $\begin{array}{l}\text { Lissencephaly (pachygyria), } \\
\text { Cerebral, and cerebellar atrophy }\end{array}$ & - & - \\
\hline 2 & - & - & Normal & Normal & - \\
\hline 3 & - & - & Hypoplasia of corpus callosum & - & - \\
\hline 4 & - & - & Mega cisterna magna & - & $\begin{array}{l}\text { Anterior fontanel closure, } \\
\text { trigonocephaly }\end{array}$ \\
\hline 5 & - & $\begin{array}{l}\text { Anterior and posterior } \\
\text { fontanel closure, } \\
\text { craniosynostosis }\end{array}$ & Normal & - & Microcephaly \\
\hline 6 & - & - & Normal & - & - \\
\hline 7 & - & - & $\begin{array}{l}\text { Intracranial hemorrhage, } \\
\text { hydrocephalus }\end{array}$ & - & - \\
\hline 8 & - & Hydrocephalus & $\begin{array}{l}\text { Intracranial hemorrhage, } \\
\text { hydrocephalus, hypoplasia } \\
\text { of the corpus callosum }\end{array}$ & - & - \\
\hline 9 & - & - & $\begin{array}{l}\text { Hydrocephalus, hypoplasia } \\
\text { of the corpus callosum }\end{array}$ & - & - \\
\hline 10 & - & $\begin{array}{l}\text { Diffuse periventricular } \\
\text { calcifications, } \\
\text { lissencephaly }\end{array}$ & Lissencephaly & - & - \\
\hline 11 & - & - & $\begin{array}{l}\text { Lissencephaly (pachygyria), } \\
\text { colpocephaly }\end{array}$ & - & - \\
\hline 12 & - & - & Normal & - & - \\
\hline 13 & - & - & Normal & - & - \\
\hline 14 & - & - & $\begin{array}{l}\text { Colpocephaly, hypoplasia } \\
\text { of the corpus callosum }\end{array}$ & - & - \\
\hline 15 & - & - & Chiari malformation type 2 & - & - \\
\hline 16 & - & $\begin{array}{l}\text { Periventricular } \\
\text { leukomalacia }\end{array}$ & - & - & Craniosynostosis \\
\hline 17 & - & $\begin{array}{l}\text { Intracranial } \\
\text { hemorrhage }\end{array}$ & - & - & - \\
\hline 18 & - & - & Delayed myelination & - & - \\
\hline 19 & $\begin{array}{l}\text { Periventricular } \\
\text { calcifications }\end{array}$ & - & - & - & - \\
\hline 20 & - & $\begin{array}{l}\text { Linear periventricular } \\
\text { calcifications }\end{array}$ & - & - & - \\
\hline 21 & - & $\begin{array}{l}\text { Diffuse periventricular } \\
\text { calcifications }\end{array}$ & - & - & - \\
\hline 22 & - & $\begin{array}{l}\text { Periventricular } \\
\text { calcifications }\end{array}$ & - & - & - \\
\hline 23 & $\begin{array}{l}\text { Periventricular } \\
\text { calcifications }\end{array}$ & - & - & - & - \\
\hline 24 & - & - & $\begin{array}{l}\text { Delayed myelination, intracranial } \\
\text { hemorrhage, hydrocephalus }\end{array}$ & - & - \\
\hline
\end{tabular}

Abbreviations: CMV, cytomegalovirus; CT, computed tomography; MRI, magnetic resonance imaging; US, ultrasound.

\section{Discussion}

Cytomegalovirus is the most common pathogen leading to congenital infections worldwide. CMV infection may occur by in utero transmission, primary maternal infection, reactiva- tion, or during reinfection of seropositive mothers. ${ }^{8}$ If a mother has primary CMV infection during pregnancy, it is likely to cause a severe damage in fetus and it is easy to diagnose in presence of clinical findings. Nonetheless, viral shedding continues for years after the primary infection among children, ${ }^{9}$ 

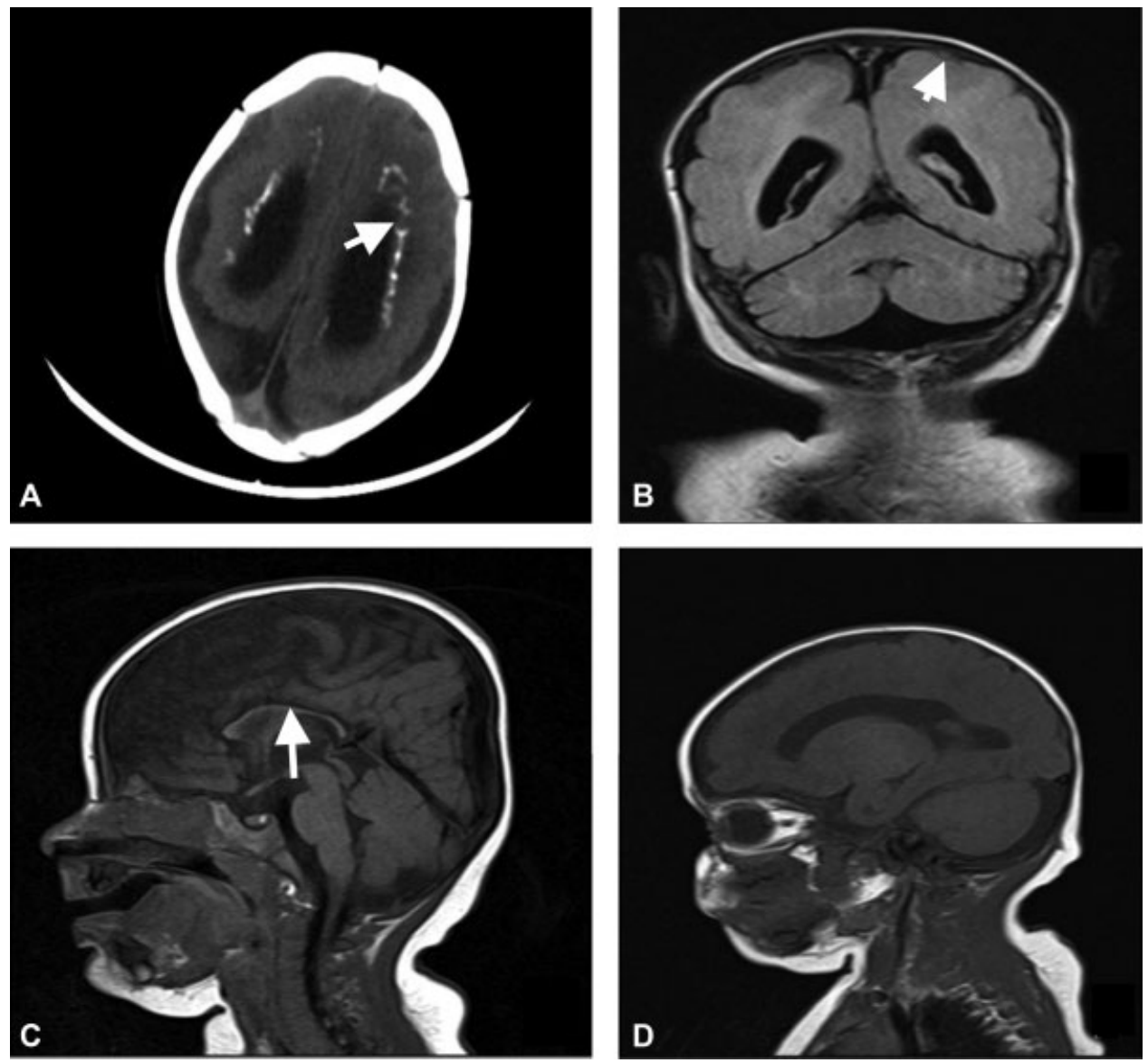

Fig. 1 MRI features of congenital and possible congenital CMV infection cases. (A): Case 10; bilateral periventriculer calcifications (B and D): Case 1; lissencephaly (pachygyria) (C): Case 3; hypoplasia of the corpus callosum. CMV, Cytomegalovirus; MRI, magnetic resonance imaging.
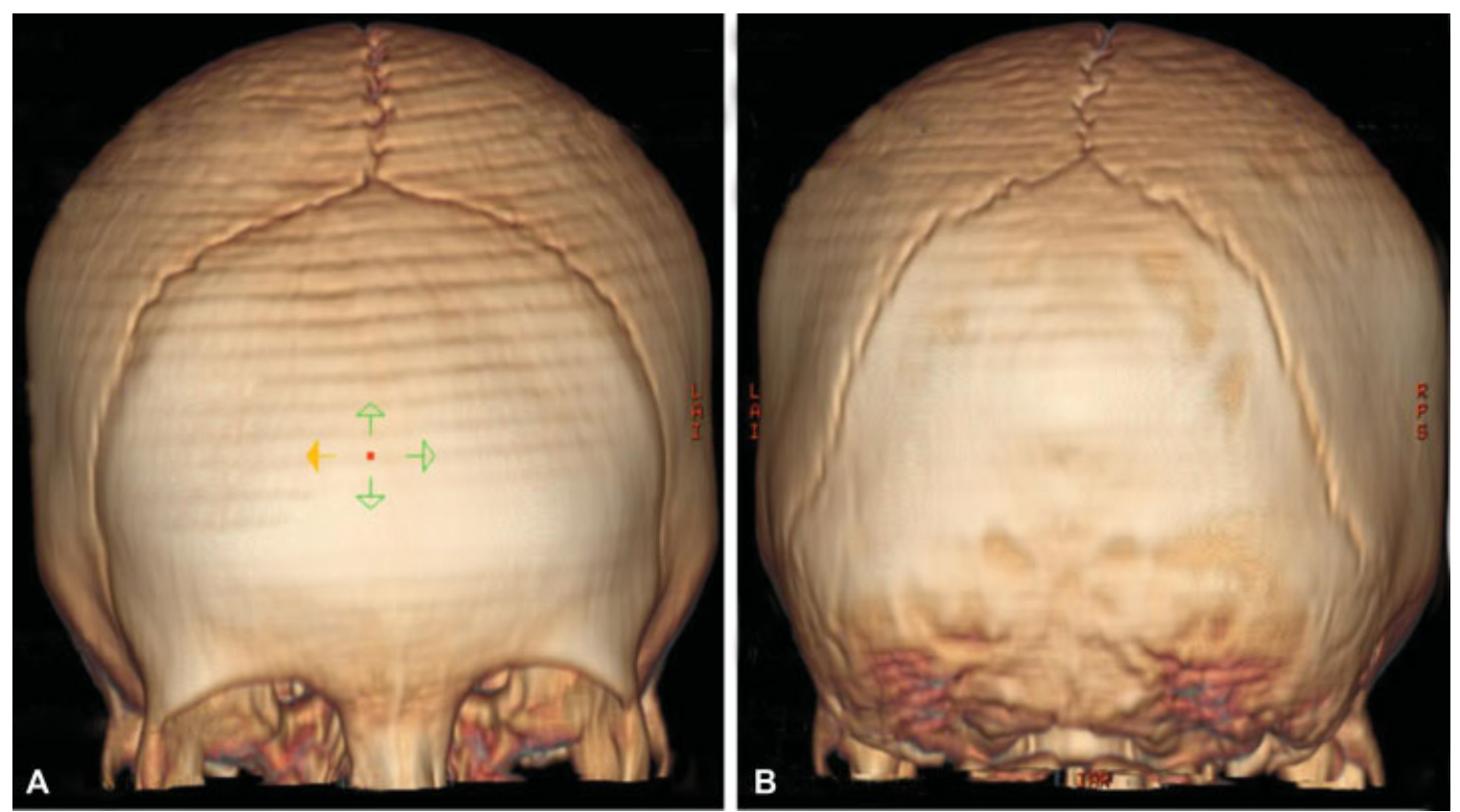

Fig. 2 Three-dimensional CT features of case no. 5; microcephaly and craniosynostosis. CT, computed tomography.

and sings of infections may appear at older ages. In this study, patients who had no clinical findings during neonatal period but had different clinical presentation before 1 year of age were diagnosed with possible cCMV infection in accordance with diagnostic criteria and it was observed to be more common compared with the cCMV infection.

Congenital CMV infection is mostly asymptomatic. However, in symptomatic infection, petechia is the most common 
symptom with a rate of 54 to $76 \%{ }^{7}$ Furthermore, symptoms, such as microcephaly, small size for gestational age (SGA), hepatomegaly, splenomegaly, chorioretinitis, and loss of vision may also be observed. ${ }^{10-13}$ In our study, petechia was the most common symptom in patients with congenital CMV infection, as is in the literature. Microcephaly has been reported in half of the patients with symptomatic cCMV infection. ${ }^{14}$ In our study, however, other life-threatening clinical findings were at more forefronts in patients with cCMV infection. Yet, microcephaly was the most common symptom in our patients with possible CCMV infection. A possible reason for this is that patients with asymptomatic cCMV infection could not be diagnosed within the 1st weeks after birth and, consequently, these patients were diagnosed with possible cCMV infection when they were admitted to the clinic due to development of microcephaly.

It is known that $90 \%$ of the patients with symptomatic CCMV infection will develop neurological sequelae and the mortality rate may vary between 5 to $30 \% .^{9}$ In this study, three (50\%) of the six cases of cCMV infection died during neonatal period. Presence of accompanying medical problems, such as prematurity and respiratory distress syndrome (RDS), in addition to cCMV infection in these patients was suggested to cause higher mortality rate in this study compared with the literature.

Cerebral involvement should be investigated by using neuroimaging methods in neonates with CCMV or possible cCMV infections. Mechanism of brain injury in congenital $\mathrm{CMV}$ is diverse. Some researchers thinking that the virus is neurotrophic and cytopathic proposed that the infection causes direct neuronal injury, leading to hypocampal, cerebellar hypoplasia, and cortical dysplasia during early phase of pregnancy (during development of the germinal matrix). ${ }^{15}$ Additionally, CMV may cause vasculitis in cerebral vessels, infecting the endothelial cells. ${ }^{16-18}$ Prevalence of cortical malformations, ventriculomegaly, and hypocampal dysplasia in brain MRI images of patients with congenital CMV has been increased. ${ }^{19}$ In addition to this, dilated lateral ventricles, oligo/pachygyria, pathological mineralization, paraventricular cysts, and intracranial calcifications were also detected in these patients. ${ }^{20,21}$ Van der Knaap et al showed in their study that multifocal white matter lesions involving deep parietal white matter were present in patients with cCMV infection, regardless of presence of gyral abnormality. ${ }^{22}$ In our study, the most commonly detected findings after MR images were hypoplasia of corpus callosum and hydrocephalous. These were followed by lissencephaly and intracranial hemorrhage. Abnormal MR images were more common in patients with possible cCMV infection compared with patients with CCMV infection. We think that the early death of patients with cCMV infection did not allow to perform neuroimaging studies.

Association of abnormal neuroimaging findings with neurodevelopmental sequelae in long-term follow-up has been shown in previous studies. ${ }^{23}$ We also, similarly, observed neurodevelopmental sequelae, such as developmental motor delay, seizures, and cognitive disorder in all patients in our study. Normal neuroimaging characteristics at birth present good long-term neurological outcomes. ${ }^{13}$ In this study, we observed poor neurodevelopmental outcomes also in patients with normal MR image. We think, although MR image was normal in these patients, concomitance of seizure or craniosynostosisinduced microcephaly influenced the prognosis negatively.

Early diagnosis of asymptomatic cCMV infection is difficult and, in fact, CMV infection continues to damage the developing brain, even if it is asymptomatic. In this study, we observed that the patients were being followed-up puzzlingly with different complaints and diagnoses. This study is important as it is the first study evaluating clinical and neuroimaging outcomes of possible cCMV infection. The limitation of the study is its small sample size, being retrospective, and additionally insufficient neuroimaging of especially patients with cCMV.

\section{Conclusion}

This study suggests that possible cCMV infections are more prevalent compared with cCMV infections and delays in diagnosis occur due to not keeping suspicion of CMV infection in early phase. Clinicians should consider long-term neurodevelopmental sequelae of cCMV and possible cCMV infections and remember to screen congenital CMV infection in suspected individuals.

Conflict of Interest

None declared.

\section{References}

1 Picone O, Vauloup-Fellous C, Cordier AG, et al. A series of 238 cytomegalovirus primary infections during pregnancy: description and outcome. Prenat Diagn 2013;33(08):751-758

2 Syggelou A, Iacovidou N, Kloudas S, Christoni Z, Papaevangelou V. Congenital cytomegalovirus infection. Ann NY Acad Sci 2010; 1205:144-147

3 Fowler KB, Boppana SB. Congenital cytomegalovirus infection. Semin Perinatol 2018;42(03):149-154

4 Kwak M, Yum MS, Yeh HR, Kim HJ, Ko TS. Brain magnetic resonance imaging findings of congenital cytomegalovirus infection as a prognostic factor for neurological outcome. Pediatr Neurol 2018;83:14-18

5 Noyola DE, Demmler GJ, Nelson CT, et al; Houston Congenital CMV Longitudinal Study Group. Early predictors of neurodevelopmental outcome in symptomatic congenital cytomegalovirus infection. J Pediatr 2001;138(03):325-331

6 Bartlett AW, McMullan B, Rawlinson WD, Palasanthiran P. Hearing and neurodevelopmental outcomes for children with asymptomatic congenital cytomegalovirus infection: a systematic review. Rev Med Virol2017. doi: 10.1002/rmv.1938

7 Demmler-Harrison GJ. Congenital cytomegalovirus infection: clinical features and diagnosis. Available at: https://www.uptodate. com/contents/congenital-cytomegalovirus-infection-clinical-features-and-diagnosis. Accessed May 24, 2018

8 Munro SC, Hall B, Whybin LR, et al. Diagnosis of and screening for cytomegalovirus infection in pregnant women. J Clin Microbiol 2005;43(09):4713-4718

9 Coll O, Benoist G, Ville Y, et al; WAPM Perinatal Infections Working Group. Guidelines on CMV congenital infection. J Perinat Med 2009;37(05):433-445

10 Boppana SB, Ross SA, Fowler KB. Congenital cytomegalovirus infection: clinical outcome. Clin Infect Dis 2013;57(4, Suppl 4): S178-S181 
11 Kylat RI, Kelly EN, Ford-Jones EL. Clinical findings and adverse outcome in neonates with symptomatic congenital cytomegalovirus (SCCMV) infection. Eur J Pediatr 2006;165(11):773-778

12 Dreher AM, Arora N, Fowler KB, et al. Spectrum of disease and outcome in children with symptomatic congenital cytomegalovirus infection. J Pediatr 2014;164(04):855-859

13 Buonsenso D, Serranti D, Gargiullo L, Ceccarelli M, Ranno O, Valentini P. Congenital cytomegalovirus infection: current strategies and future perspectives. Eur Rev Med Pharmacol Sci 2012; 16(07):919-935

14 Williamson WD, Desmond MM, LaFevers N, Taber LH, Catlin FI, Weaver TG. Symptomatic congenital cytomegalovirus. Disorders of language, learning, and hearing. Am J Dis Child 1982;136(10): 902-905

15 Barkovich AJ, Lindan CE. Congenital cytomegalovirus infection of the brain: imaging analysis and embryologic considerations. Am J Neuroradiol 1994;15(04):703-715

16 Nigro G, La Torre R, Sali E, et al. Intraventricular haemorrhage in a fetus with cerebral cytomegalovirus infection. Prenat Diagn 2002; 22(07):558-561

17 Persoons MC, Stals FS, van dam Mieras MC, Bruggeman CA. Multiple organ involvement during experimental cytomegalovirus infection is associated with disseminated vascular pathology. J Pathol 1998; 184(01):103-109

18 Golden MP, Hammer SM, Wanke CA, Albrecht MA. Cytomegalovirus vasculitis. Case reports and review of the literature. Medicine (Baltimore) 1994;73(05):246-255

19 Manara R, Balao L, Baracchini C, Drigo P, D’Elia R, Ruga EM. Brain magnetic resonance findings in symptomatic congenital cytomegalovirus infection. Pediatr Radiol 2011;41(08):962-970

20 Boesch C, Issakainen J, Kewitz G, Kikinis R, Martin E, Boltshauser E. Magnetic resonance imaging of the brain in congenital cytomegalovirus infection. Pediatr Radiol 1989;19(02):91-93

21 Oosterom N, Nijman J, Gunkel J, et al. Neuro-imaging findings in infants with congenital cytomegalovirus infection: relation to trimester of infection. Neonatology 2015;107(04):289-296

22 van der Knaap MS, Vermeulen G, Barkhof F, Hart AA, Loeber JG, Weel JF. Pattern of white matter abnormalities at MR imaging: use of polymerase chain reaction testing of Guthrie cards to link pattern with congenital cytomegalovirus infection. Radiology 2004;230(02):529-536

23 Suzuki Y, Toribe Y, Mogami Y, Yanagihara K, Nishikawa M. Epilepsy in patients with congenital cytomegalovirus infection. Brain Dev 2008;30(06):420-424 